\section{Getting down to the bare bones}

\section{Richard Potts}

The Analysis of Animal Bones from Archeological Sites. By Richard G. Klein and Kathryn Cruz-Uribe. University of Chicago Press: 1984. Pp.266. Hbk \$19.95, £18.95: pbk \$8.95, $£ 8.50$.

INVESTIGATION of past human activities and ecological settings requires many analytical steps and cautious weighing of the processes that transform living phenomena into buried, fossilized traces Archaeological faunal remains - one such "end product" of this transformationhave provided extremely important, albeit controversial, evidence about early hominid behaviour and environments After determining the species, body part, sex and age of death of the animals represented by bone specimens, zooarchaeologists often attempt to estimate the relative number of individuals or bone units of each species found in an accumulation of bones; further, they may construct mortality/age distributions for wellrepresented species. These and other quantitative data help to test ideas about environmental change; catastrophic versus attritional death of animals; whether humans, carnivores or geological agents were responsible for bone aggregates; and whether humans hunted or scavenged, and how they selected certain species and body parts.

The theme of this volume is the entire process by which zooarchaeologists interpret the past from the assemblages of vertebrate bones often found

\section{The wonderful sky at night}

Martin Harwit

The Cambridge Atlas of Astronomy. Edited by Jean Audouze and Guy Isräel. Cambridge University Press: 1985. Pp.432. $£ 29.95, \$ 75$.

How wonderful to open this volume to find an absolutely lavish collection of new drawings, charts, explanatory diagrams. illustrated tables and photographs - not just of astronomical objects, but also of terrestrial fossils and atmospheric and volcanic structures

There are charts and maps of all kinds, and pictures of the lunar surface and of all the planets and satellites visited by spacecraft. Some of these we have seen before, but most of them are selected to be instructive: tracks left on the Moon's surface by the Apollo lunar vehicle, along archaeological excavations. The first of two sections introduces lucidly the fundamentals of animal bone identification, faunal data collection and analysis. The second provides computer programs in BASIC that generate data used widely in faunal analysis (minimum number of individuals, age distributions and so on). These programs are explained clearly and the procedures used to quantify bone assemblage characteristics are discussed thoroughly. Inferences about ancient people, their sites and environments are illustrated largely from Klein's own research on prehistoric faunas of southern Africa and Europe. Thus, the book provides an excellent summary of the approaches Klein has developed.

In illustrating certain aspects of faunal analysis, the authors offer interpretations that appear post hoc, without due consideration of alternatives; this will disquiet some researchers. Others may feel that the authors' distinction between the comparative and taphonomic approaches to faunal studies is unwarranted, especially since the latter approach (which examines how modern processes create faunal patterns) is played down.

These potential points of dispute do not overshadow the book's value, however. It will be widely used as a text on fundamental aspects of faunal analysis and as a source of ideas and useful programs for generating data that help to explore the subtle, bony traces of ancient human activities and environments preserved in the geological record.

Richard Potts is Associate Curator in the De partment of Anthropology, National Museum of Natural History, Smithsonian Institution, Washington, DC 20560, USA.

My particular disappointment started when I stopped looking at the illustrations and began reading the text. In just the first hour of browsing I noted a dozen flaws of all kinds. There are numerous typographical errors, and the index appears to have been compiled with insufficient care over the cross-referencing, which is so important in a volume such as this. More specifically, a figure caption (p.11) describes the telescopes used by Galileo, and adds, "The principles of these telescopes were conceived in Venice by an unknown genius". Actually, these instruments first surfaced in the Netherlands, late in 1608 , roughly a year before Galileo began his observations. And on p. 387 we find the statement: "The existence of neutrinos was proposed for the first time by Paul Dirac on a theoretical basis to explain the conservation of the angular momentum, or spin of the ensemble of particles produced by the beta disintegration of the neutron". Actually it was Wolfgang Pauli, with some help from Enrico Fermi in naming the particle, who conceived of the neutrino for conserving energy in beta decay.

More serious, however, is the question of purpose. How much should the text explain - couldn't the occasional bit of calculus have been avoided through use of a simple diagram? One has the impression that the editors wanted to pack every possible concept into this single volume massive neutrinos, the number of neutrino generations, proton decay and other current exotica. This has taken its toll on clarity. For example the section on cosmology begins by listing five principles alleged to guide the construction of cosmological models: "The principle of uniformity, the cosmological principle, the anthropic principle, the principle of equivalence and Mach's principle" (p.381). But Mach's principle still does not fit into any of our current theoretical models; and the anthropic principle merely tells us to disregard theoretical models which don't ultimately produce cosmologists. Everything seems to have been crammed in with little regard for separating the important from the frivolous.

Still, the editors and publisher have done remarkably well with the illustrations and, on balance. I recommend the Atlas highly. I just hope that the text can be thought through once more. in time for a well-deserved second edition. ic matter and some of the processes to which both have been subjected. In turn, they will better appreciate the way a knowledge of these processes helps us to understand the past histories of the materials which make up the Solar System.

This, then, is a beautifully produced book of great pedagogic value. Amateur astronomers, high school students and professionals will find here a visual display which is both arresting and informative. What I am about to say should. therefore. not discourage one from investing in this volume.
Martin Harwit is a Professor in the Department York 14850, USA

\section{New in paperback}

- The Dawn of Animal Life: A Biohistorical Study by Martin F. Glaessner, Cambridge University Press, $£ 9.95 . \$ 19.95$

- Insroduction to Ouantum Mechanics with Applications to Chemistry by Linus Pauling and E. Bright Wilson. Jr. Dover. \$9.95 of Astronomy, Comell University, Ithaca. New 